\title{
ANALYTIC CAMPANATO SPACES AND THEIR COMPOSITIONS
}

\author{
JIE XIAO AND CHENG YUAN
}

\begin{abstract}
This paper is devoted to characterizing the analytic Campanato spaces $\mathcal{A L}_{p, \eta}$ (including the analytic Morrey spaces, the analytic John-Nirenberg space, and the analytic Lipschitz/Hölder spaces) on the complex unit disk $\mathbb{D}$ in terms of the Möbius mappings and the Littlewood-Paley forms, and consequently their compositions with the analytic self-maps of $\mathbb{D}$.
\end{abstract}

\section{CONTENTS}

1. Introduction

1.1. Background

1.2. Overview

2. Harmonic extensions of Campanato spaces

2.1. Representation via Möbius transforms

2.2. Littlewood-Paley type characters

3. Analytic Campanato spaces in Bloch type spaces 15

3.1. Generalized Carleson measures 15

3.2. Distance estimates

4. Compositions for analytic Campanato spaces 17

4.1. Actions between analytic Campanato spaces 17

4.2. Actions between analytic Campanato and Bloch type spaces 21

References 22

\section{INTRODUCTION}

1.1. Background. In partial differential equations and harmonic analysis over the unit circle $\mathbb{T}$ of the complex plane $\mathbb{C}$, the Campanato spaces (named after S. Campanato, cf. $[2,[3]) \mathcal{L}_{p, \eta}(\mathbb{T})$ exist as an important family of spaces that are determined via certain oscillations on $\mathbb{T}$ and hence generalize the John-Nirenberg space (named after F. John and L. Nirenberg, cf. [12]) $B M O(\mathbb{T})$ (bounded mean oscillation on $\mathbb{T}$ ) and the Lipschitz spaces (named after R. Lipschitz) or the Hölder spaces (named after O. Hölder) $\operatorname{Lip}_{\alpha}(\mathbb{T})$.

To see this picture clearly, referring to [24, p. 215] we adapt the following definition of the Campanato spaces. For $(p-1, \eta) \in[0, \infty) \times[0, \infty)$ denote by $\mathcal{L}_{p, \eta}:=\mathcal{L}_{p, \eta}(\mathbb{T})$

2000 Mathematics Subject Classification. Primary 30H10, 30H25, 30H30, 30H35, 47A20, 47A25.

Key words and phrases. Analytic Campanato spaces, bounded composition operators.

Jie Xiao was supported by NSERC of Canada and URP of Memorial University. Cheng Yuan was supported by NSFC 11226086 of China and Tianjin Advanced Education Development Fund 20111005. 
the $(p, \eta)$-Campanato space of all functions $f \in L^{p}(\mathbb{T})$ with

$$
\|f\|_{p, \eta}=\sup _{I \subseteq \mathbb{T}}\left(\frac{1}{|I|^{\eta}} \int_{I}\left|f(\zeta)-f_{I}\right|^{p} \frac{|\mathrm{d} \zeta|}{2 \pi}\right)^{\frac{1}{p}}<\infty .
$$

In the above and below, $\sup _{I \subseteq \mathbb{T}}$ means the supremum taken over all subarcs $I$ of $\mathbb{T}$, and

$$
\left\{\begin{array}{l}
\zeta=e^{i t} \\
\mathrm{~d} \zeta=i e^{i t} \mathrm{~d} t \\
|I|=(2 \pi)^{-1} \int_{I}|\mathrm{~d} \zeta| \\
f_{I}=(2 \pi|I|)^{-1} \int_{I} f(\zeta)|\mathrm{d} \zeta|
\end{array}\right.
$$

It is easy to get that $\mathcal{L}_{p, \eta}$ is a Banach space with the norm

$$
\|f\|_{p}+\|f\|_{p, \eta}=\left(\int_{\mathbb{T}}|f(\zeta)|^{p} \frac{|\mathrm{d} \zeta|}{2 \pi}\right)^{\frac{1}{p}}+\|f\|_{p, \eta},
$$

and enjoys the following space monotonicity

$$
\left\{\begin{array}{l}
\infty>\eta, \lambda \geq 0 \\
\infty>p \geq q \geq 1 \\
(\eta-1) / p \geq(\lambda-1) / q
\end{array} \Longrightarrow \mathcal{L}_{p, \eta} \subseteq \mathcal{L}_{q, \lambda} .\right.
$$

Moreover, the following table shows the well-known relationship among the abovementioned function spaces (cf. [10, pp. 65-70] or [2, 3, 14, 22, 15, 12]):

\begin{tabular}{|l|l|}
\hline Index $(p, \eta)$ & Campanato space $\mathcal{L}_{p, \eta}(\mathbb{T})$ \\
\hline$\eta=0$ & Lebesgue space $L^{p}(\mathbb{T})$ \\
\hline$\eta \in(0,1)$ & Morrey space $L^{p, 1-\eta}(\mathbb{T})$ \\
\hline$\eta=1$ & John-Nirenberg space $B M O(\mathbb{T})$ \\
\hline$\eta \in(1,1+p]$ & Lipschitz/Hölder space $\operatorname{Lip}(\eta-1) / p$ \\
& $(\mathbb{T})$ \\
\hline$\eta \in(1+p, \infty)$ & Space of constants $\mathbb{C}$ \\
\hline
\end{tabular}

1.2. Overview. From the point of view of complex analysis in one variable, it is very natural to study the analytic extensions

$$
\mathcal{A L}_{p, \eta}:=\mathcal{L}_{p, \eta} \cap \text { Analytic Hardy space } H^{p}
$$

of the Campanato spaces on $\mathbb{T}$ to the open unit disk $\mathbb{D}$, where $f \in H^{p}:=H^{p}(\mathbb{T})$ if and only if $f$ is analytic in $\mathbb{D}$ and $\sup _{r \in(0,1)}\|f(r \cdot)\|_{p}<\infty$. Evidently, $\mathcal{A} \mathcal{L}_{p, \eta}$ is a Banach space under the norm $\|\cdot\|_{p}+\|\cdot\|_{p, \eta}$, and has the following structure diagram:

\begin{tabular}{|l|l|}
\hline Index $(p, \eta)$ & Analytic Campanato space $\mathcal{A L}_{p, \eta}$ \\
\hline$\eta=0$ & Analytic Hardy space $H^{p}$ \\
\hline$\eta \in(0,1)$ & Analytic Morrey space $A L^{p, 1-\eta}$ \\
\hline$\eta=1$ & Analytic John-Nirenberg space $B M O A$ \\
\hline$\eta \in(1,1+p]$ & Analytic Lipschitz/Hölder space ALip $(\eta-1) / p$ \\
\hline$\eta \in(1+p, \infty)$ & Space of constants $\mathbb{C}$ \\
\hline$p \geq q \& \frac{\eta-1}{p} \geq \frac{\lambda-1}{q}$ & $\mathcal{A L}_{p, \eta} \subseteq \mathcal{A L}_{q, \lambda}$ \\
\hline
\end{tabular}


As far as we know, the following papers [25, 27, 28, 4] have touched some aspects of these analytic Campanato spaces. Thus, such an area deserves in the highest degree to be developed further. In this paper, we will establish the following assertion:

Theorem 1. For $w \in \overline{\mathbb{D}}=\mathbb{D} \cup \mathbb{T}$ let $z \mapsto \sigma_{w}(z)=(w-z)(1-\bar{w} z)^{-1}$ be a Möbius self-map of $\overline{\mathbb{D}}$.

(i) For $0<\eta<2<1+p<\infty$, an analytic function $f$ on $\mathbb{D}$ belongs to $\mathcal{A L}_{p, \eta}$ if and only if

$$
\|f\|_{\mathcal{A} \mathcal{L}_{p, \eta, *}}:=\sup _{w \in \mathbb{D}}\left(1-|w|^{2}\right)^{\frac{1-\eta}{p}}\left\|f \circ \sigma_{w}-f(w)\right\|_{p}<\infty
$$

if and only if

$$
\|f\|_{\mathcal{A} \mathcal{L}_{p, \eta, \star}}:=\sup _{I \subseteq \mathbb{T}}\left(\frac{1}{|I|^{\eta}} \int_{I}\left(\int_{1-|I|}^{1}\left|f^{\prime}(r \zeta)\right|^{2}(1-r) \mathrm{d} r\right)^{\frac{p}{2}} \frac{|\mathrm{d} \zeta|}{2 \pi}\right)^{\frac{1}{p}}<\infty .
$$

(ii) For $0<\eta \leq 1+p<\infty, 1<p<\infty$ and $\alpha=(p+1-\eta) / p$, $\mathcal{A L}_{p, \eta}$ is contained in the Bloch type space

$$
\mathcal{B}_{\alpha}=\left\{f: f \text { is analytic in } \mathbb{D} \text { with }\|f\|_{\mathcal{B}_{\alpha}}:=\sup _{w \in \mathbb{D}}\left(1-|w|^{2}\right)^{\alpha}\left|f^{\prime}(w)\right|<\infty\right\} .
$$

In particular, for $p=2$ and $f \in \mathcal{B}_{\alpha}$, one has

$$
\inf _{g \in \mathcal{A} \mathcal{L}_{p, \eta}}\|f-g\|_{\mathcal{B}_{\alpha}}=\inf \left\{\varepsilon: \chi_{\Omega_{\varepsilon}(f)}\left(1-|z|^{2}\right)^{\eta-2} \mathrm{~d} A(z) \text { is in } \mathcal{C} \mathcal{M}_{\eta}\right\} \text {, }
$$

where

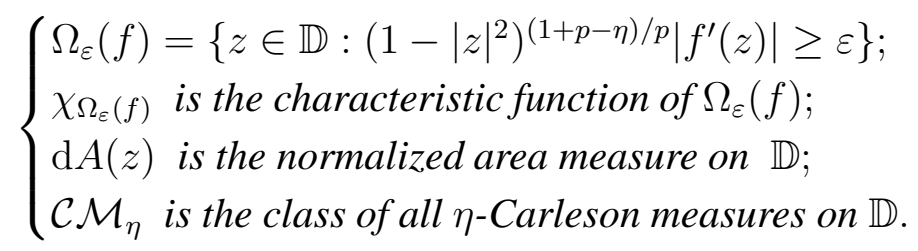

(iii) For $0<\eta, \lambda<2=q \leq p<\infty$, an analytic self-map $\varphi$ of $\mathbb{D}$, and the analytic composition $C_{\varphi} f=f \circ \varphi$, one has:

$$
\left\|C_{\varphi} f\right\|_{\mathcal{A} \mathcal{L}_{q, \lambda, *}} \lesssim\|f\|_{\mathcal{A L} p, \eta, *} \quad \forall \quad f \in \mathcal{A L}_{p, \eta}
$$

if and only if

$$
\sup _{w \in \mathbb{D}} \frac{\left(1-|w|^{2}\right)^{(1-\lambda) / q}}{\left(1-|\varphi(w)|^{2}\right)^{(1-\eta) / p}}\left\|\sigma_{\varphi(w)} \circ \varphi \circ \sigma_{w}\right\|_{q}<\infty .
$$

(iv) For $0<\eta<1+p<\infty, 0<\alpha, p-1<\infty$, an analytic self-map $\varphi$ of $\mathbb{D}$, and the analytic composition $C_{\varphi} f=f \circ \varphi$, one has:

$$
\left\|C_{\varphi} f\right\|_{\mathcal{B}_{\alpha}} \lesssim\|f\|_{\mathcal{A} \mathcal{L}_{p, \eta, \star}} \quad \forall \quad f \in \mathcal{A} \mathcal{L}_{p, \eta}
$$

respectively

$$
\left\|C_{\varphi} f\right\|_{\mathcal{A L}_{p, \eta, \star}} \lesssim\|f\|_{\mathcal{B}_{\alpha}} \quad \forall \quad f \in \mathcal{B}_{\alpha}
$$

if and only if

$$
\sup _{w \in \mathbb{D}} \frac{\left(1-|w|^{2}\right)^{\alpha}\left|\varphi^{\prime}(w)\right|}{\left(1-|\varphi(w)|^{2}\right)^{\frac{p+1-\eta}{p}}}<\infty
$$


respectively

$$
\sup _{I \subseteq \mathbb{T}}\left(\frac{1}{|I|^{\eta}} \int_{I}\left(\int_{1-|I|}^{1}\left|\varphi^{\prime}(r \zeta)\right|^{2}(1-r)^{1-2 \alpha} \mathrm{d} r\right)^{\frac{p}{2}} \frac{|\mathrm{d} \zeta|}{2 \pi}\right)^{\frac{1}{p}}<\infty .
$$

Notation. As we go along with proving Theorem 1 in $\$ 2-83-84$, we will introduce the required notation. But here, we only use $U \lesssim V$ (or $V \gtrsim U$ ) as $U \leq c V$ for a positive constant $c$, and moreover write $U \approx V$ for both $U \lesssim V$ and $V \lesssim U$.

\section{HARMONIC EXTENSIONS OF CAMPANATO SPACES}

2.1. Representation via Möbius transforms. For $f \in L^{1}(\mathbb{T})$ and $z \in \mathbb{D}$, let $P_{z}(\zeta)=$ $\left(1-|z|^{2}\right) /|\zeta-z|^{2}$ be the Poisson kernel at $z$ and $P f$ be the Poisson extension of $f$, that is

$$
P f(z)=\frac{1}{2 \pi} \int_{\mathbb{T}} f(\zeta) P_{z}(\zeta)|\mathrm{d} \zeta|=\frac{1}{2 \pi} \int_{0}^{2 \pi} \Re\left(\frac{e^{i \theta}+z}{e^{i \theta}-z}\right) f\left(e^{i \theta}\right) \mathrm{d} \theta .
$$

As an extension of Theorem 3.2.1 in [27], we have the following Möbius type representation of a Campanato space, whence reaching the first part of Theorem[1(i).

Theorem 2. Let $0<\eta<2 \leq 1+p<\infty$.

(i) For an $L^{p}$ function $f, f \in \mathcal{L}_{p, \eta}$ if and only if

$$
\|f\|_{p, \eta, *}:=\sup _{w \in \mathbb{D}}\left(\left(1-|w|^{2}\right)^{1-\eta} \int_{\mathbb{T}}\left|f \circ \sigma_{w}(\zeta)-P f(w)\right|^{p}|\mathrm{~d} \zeta|\right)^{\frac{1}{p}}<\infty .
$$

(ii) For an $H^{p}$ function $f, f \in \mathcal{A} \mathcal{L}_{p, \eta}$ if and only if $\|f\|_{\mathcal{A L}_{p, \eta, *}}<\infty$.

Proof. (i) Suppose $f \in \mathcal{L}_{p, \eta}$. For each nonzero $w \in \mathbb{D}$, let $I_{w}$ be the subarc of $\mathbb{T}$ with center $w /|w|$ and length $1-|w|$; and for $w=0$, let $I_{w}=\mathbb{T}$. Moreover, for $j=0,1, \ldots, n-1$, let $J_{j}=2^{j} I_{w}$, where $n$ is the smallest natural number such that $2^{n}\left|I_{w}\right| \geq 1$. Obviously, we may assume $J_{n}=\mathbb{T}$. Under this circumstance, we have that for a given point $w \in \mathbb{D}$,

$$
\begin{aligned}
& \int_{\mathbb{T}}|f(\zeta)-P f(w)|^{p} \frac{1-|w|^{2}}{|\zeta-w|^{2}}|\mathrm{~d} \zeta| \\
& \lesssim \int_{\mathbb{T}}\left|f(\zeta)-f_{I_{w}}\right|^{p} \frac{1-|w|^{2}}{|\zeta-w|^{2}}|\mathrm{~d} \zeta| \\
& \lesssim\left(\int_{J_{0}}+\sum_{j=0}^{n-1} \int_{J_{j+1} \backslash J_{j}}\right)\left|f(\zeta)-f_{I_{w}}\right|^{1} \frac{1-|w|^{2}}{|\zeta-w|^{2}}|\mathrm{~d} \zeta| \\
& \lesssim\left(1-|w|^{2}\right)^{-1}\left(\int_{J_{0}}+\sum_{j=0}^{n-1} 2^{-2 j} \int_{J_{j+1} \backslash J_{j}}\right)\left|f(\zeta)-f_{I_{w}}\right|^{p}|\mathrm{~d} \zeta| \\
& \lesssim \frac{1}{\left|J_{0}\right|} \int_{J_{0}}\left|f(\zeta)-f_{I_{w}}\right|^{p}|\mathrm{~d} \zeta|+\sum_{j=0}^{n-1} \frac{2^{-j}}{\left|J_{j+1}\right|} \int_{J_{j+1} \backslash J_{j}}\left|f(\zeta)-f_{I_{w}}\right|^{p}|\mathrm{~d} \zeta| .
\end{aligned}
$$


Using Hölder's inequality and $f \in \mathcal{L}_{p, \eta}$, we get

$$
\begin{aligned}
& \left|f_{J_{j+1}}-f_{J_{j}}\right| \\
& \lesssim \frac{1}{\left|J_{j+1}\right|} \int_{J_{j+1}}\left|f(\zeta)-f_{I_{w}}\right||\mathrm{d} \zeta| \\
& \lesssim\left(\frac{2}{\left|J_{j+1}\right|} \int_{J_{j+1}}\left|f(\zeta)-f_{I_{w}}\right|^{p}|\mathrm{~d} \zeta|\right)^{\frac{1}{p}} \\
& \lesssim\left|J_{j+1}\right|^{\frac{\eta-1}{p}}\|f\|_{p, \eta} \\
& \lesssim 2^{(\eta-1)(j+1) / p}(1-|w|)^{\frac{\eta-1}{p}}\|f\|_{p, \eta},
\end{aligned}
$$

whence reaching

$$
\begin{aligned}
& \left|f_{J_{j+1}}-f_{J_{0}}\right| \\
& \lesssim\left|f_{J_{j+1}}-f_{J_{j}}\right|+\cdots+\left|f_{J_{1}}-f_{J_{0}}\right| \\
& \lesssim j\left(1+2^{j(\eta-1) / p}\right)(1-|w|)^{\frac{\eta-1}{p}}\|f\|_{p, \eta} .
\end{aligned}
$$

Accordingly,

$$
\begin{aligned}
& \frac{1}{\left|J_{j+1}\right|} \int_{J_{j+1}}\left|f(\zeta)-f_{J_{0}}\right|^{p}|\mathrm{~d} \zeta| \\
& \leq\left(\left(\frac{1}{\left|J_{j+1}\right|} \int_{J_{j+1}}\left|f(\zeta)-f_{J_{j+1}}\right|^{p}|\mathrm{~d} \zeta|\right)^{1 / p}+\left|f_{J_{j+1}}-f_{J_{0}}\right|\right)^{p} \\
& \lesssim j^{p}\left(1+2^{j(\eta-1)}\right)(1-|w|)^{\eta-1}\|f\|_{p, \eta}^{p}
\end{aligned}
$$

Putting the above estimates together, we obtain

$$
\begin{aligned}
& \int_{\mathbb{T}}\left|f \circ \sigma_{w}(\zeta)-P f(w)\right|^{p}|\mathrm{~d} \zeta| \\
& \approx \int_{\mathbb{T}}|f(\zeta)-P f(w)|^{p} \frac{1-|w|^{2}}{|\zeta-w|^{2}}|\mathrm{~d} \zeta| \\
& \lesssim(1-|w|)^{\eta-1}\|f\|_{p, \eta}^{p} \sum_{j \geq 0} 2^{-j} j^{p}\left(1+2^{j(\eta-1)}\right) .
\end{aligned}
$$

Since $0<\eta<2 \leq 1+p$, we have $\|f\|_{p, \eta, *} \lesssim\|f\|_{p, \eta}$.

Conversely, if $\|f\|_{p, \eta, *}<\infty$, then for any subarc $I \subseteq \mathbb{T}$, we take such a $w \in \mathbb{D}$ that $w /|w|$ is the center of $I$ and $|w|=1-|I|$, to get

$$
|\zeta-w| \lesssim 1-|w|^{2} \quad \forall \quad \zeta \in I
$$


and then

$$
\begin{aligned}
& \frac{1}{|I|^{\eta}} \int_{I}\left|f(\zeta)-f_{I}\right|^{p}|\mathrm{~d} \zeta| \\
& \lesssim \frac{1}{|I|^{\eta}} \int_{I}|f(\zeta)-P f(w)|^{p}|\mathrm{~d} \zeta| \\
& \lesssim\left(1-|w|^{2}\right)^{1-\eta} \int_{I}|f(\zeta)-P f(w)|^{p} \frac{1-|w|^{2}}{|\zeta-w|^{2}}|\mathrm{~d} \zeta| \\
& \lesssim\|f\|_{p, \eta, *}^{p},
\end{aligned}
$$

as desired.

(ii) This follows from (i) and the fact that that $P f=f$ holds for all $f \in H^{p}$.

Given $z \in \mathbb{D}$ and $1<p<\infty$. Upon writing the Szegö projection $S$ of $f \in L^{p}(\mathbb{T})$ :

$$
S f(z)=\frac{1}{2 \pi} \int_{0}^{2 \pi} \frac{f\left(e^{i \theta}\right)}{1-z e^{-i \theta}} \mathrm{d} \theta,
$$

and $\tilde{f}$ the conjugate operator of $f$ :

$$
\left\{\begin{array}{l}
\tilde{f}(z)=\frac{1}{2 \pi} \int_{0}^{2 \pi} \Im\left(\frac{e^{i \theta}+z}{e^{i \theta}-z}\right) f\left(e^{i \theta}\right) \mathrm{d} \theta \\
\text { with } \\
\tilde{f}\left(e^{i \theta}\right)=\lim _{r \rightarrow 1^{-}} \tilde{f}\left(r e^{i \theta}\right)
\end{array}\right.
$$

one has

$$
i \tilde{f}(z)+\operatorname{Pf}(z)=2 S f(z)-P f(0)
$$

Closely related to Peetre's [17, Theorem 1.1] (cf. [18]), the following result extends the $B M O$ space case (see [30, p. 272]) and the Lipschitz space case (Privalov's theorem, see [24, p. 214]).

Corollary 3. Let $0<\eta<2<1+p<\infty$. The Szegö projection $S$ maps $\mathcal{L}_{p, \eta}$ boundedly onto $\mathcal{A L}_{p, \eta}$. Equivalently, the conjugate operator is bounded on $\mathcal{L}_{p, \eta}$.

Proof. For $f \in \mathcal{L}_{p, \eta} \subset L^{p}$, write the Szegö projection of $f$ as $S(f)=u+i v$, where $u$ and $v$ are the real and imaginary parts respectively. Then

$$
(S f) \circ \sigma_{w}=u \circ \sigma_{w}+i v \circ \sigma_{w} .
$$

This means that $u$ is the Poisson extension of $f$. According to the well known result: $L^{p}$ boundedness of $S: L^{p} \rightarrow H^{p}$ when $1<p<\infty$ (see, e.g. Theorem 9.6 in [30]), we have

$$
\left\|S f \circ \sigma_{w}-S f(w)\right\|_{p} \lesssim\left\|u \circ \sigma_{w}-u(w)\right\|_{p}
$$

Thus

$$
\left(1-|w|^{2}\right)^{1-\eta}\left\|S f \circ \sigma_{w}-S f(w)\right\|_{p}^{p} \lesssim\left(1-|w|^{2}\right)^{1-\eta}\left\|u \circ \sigma_{w}-u(w)\right\|_{p}^{p}
$$


Note that $\left.u\right|_{\mathbb{T}}=f$, then $S f \in \mathcal{A} \mathcal{L}_{p, \eta}$ thanks to Theorem 1 (i). So one gets

$$
\begin{aligned}
& \left(1-|w|^{2}\right)^{1-\eta}\left\|v \circ \sigma_{w}-v(w)\right\|_{p}^{p} \\
& \lesssim\left(1-|w|^{2}\right)^{1-\eta}\left\|S f \circ \sigma_{w}-S f(w)\right\|_{p}^{p} \\
& \lesssim\|u\|_{p, \eta, *}^{p} \\
& \approx\|f\|_{p, \eta, *}^{p}
\end{aligned}
$$

This completes the proof.

2.2. Littlewood-Paley type characters. For $\eta \in(0,1]$ and $1 \leq p<\infty$, let $p^{\prime}=$ $p /(p-1)$ with convention $1^{\prime}=1 /(1-1)=\infty$. A function $a$ on $\mathbb{T}$ is called $\left(p^{\prime}, \eta\right)$-atom provided that there is a subarc $I \subseteq \mathbb{T}$ such that

$$
\left\{\begin{array}{l}
\operatorname{supp} a \subset I \\
\int_{\mathbb{T}} a(\zeta)|\mathrm{d} \zeta|=0 \\
\|a\|_{p^{\prime}} \leq|I|^{-\eta / p}
\end{array}\right.
$$

Also, let $\mathcal{H}_{p^{\prime}, \eta}:=\mathcal{H}_{p^{\prime}, \eta}(\mathbb{T})$ be the space of all functions

$$
g(\zeta)=\sum_{j=0}^{\infty} \lambda_{j} a_{j}(\zeta) \quad \text { in the sense of distribution, }
$$

where $\lambda_{j} \in \mathbb{C}, a_{j}$ is a $\left(p^{\prime}, \eta\right)$-atom and $\sum_{j=0}^{\infty}\left|\lambda_{j}\right|<\infty$.

The following result is known; see also [31, Proposition 5] and its references including [8].

\section{Lemma 4.}

(i) If $0<\eta<1<p<\infty$, then $\mathcal{L}_{p, \eta}$ is isomorphic to the dual space $\mathcal{H}_{p^{\prime}, \eta}^{*}$ under the pairing

$$
\int_{\mathbb{T}} f(\zeta) \overline{g(\zeta)} \frac{|\mathrm{d} \zeta|}{2 \pi} \quad \forall \quad(f, g) \in \mathcal{L}_{p, \eta} \times \mathcal{H}_{p^{\prime}, \eta} .
$$

(ii) If $\eta=1 \leq p<\infty$, then $\mathcal{L}_{p, \eta}=B M O$ is isomorphic to the dual space $\mathcal{H}_{\infty, 1}^{*}$ under the pairing

$$
\int_{\mathbb{T}} f(\zeta) \overline{g(\zeta)} \frac{|\mathrm{d} \zeta|}{2 \pi} \quad \forall \quad(f, g) \in B M O \times \mathcal{H}_{\infty, 1} .
$$

Inspired by [6, 23, 16, 7] we have the following equivalent characterization of the Campanato-Morrey spaces, thereby arriving at the second part of Theorem 11(i).

Theorem 5. Let

$$
\left\{\begin{array}{l}
\nabla=\left(\partial_{z}+\partial_{\bar{z}}, i\left(\partial_{z}-\partial_{\bar{z}}\right)\right) \\
1<p<\infty \\
\eta \in(0,1+p)
\end{array}\right.
$$

(i) For an $L^{p}$ function $f, f \in \mathcal{L}_{p, \eta}$ if and only if

$$
\|f\|_{p, \eta, \star}:=\sup _{I \subseteq \mathbb{T}}\left(\frac{1}{|I|^{\eta}} \int_{I}\left(\int_{1-|I|}^{1}|\nabla \operatorname{Pf}(r \zeta)|^{2}\left(1-r^{2}\right) \mathrm{d} r\right)^{\frac{p}{2}} \frac{|\mathrm{d} \zeta|}{2 \pi}\right)^{\frac{1}{p}}<\infty .
$$

(ii) For an $H^{p}$ function $f, f \in \mathcal{A} \mathcal{L}_{p, \eta}$ if and only if $\|f\|_{\mathcal{A} \mathcal{L}_{p, \eta, \star}}<\infty$. 
Proof. It suffices to verify (i). We first prove the "only if" part. If $f \in \mathcal{L}_{p, \eta}$, then any given subarc $I \subseteq \mathbb{T}$ is used to decompose $f$ via

$$
f=f_{I}+\left(f-f_{I}\right) \chi_{2 I}+\left(f-f_{I}\right) \chi_{\mathbb{T} \backslash 2 I}=f_{1}+f_{2}+f_{3}
$$

where $\chi_{J}$ is the characteristic function of $J \subseteq \mathbb{T}, 2 I \subseteq \mathbb{T}$ is the concentric double of $I$.

In sake of convenience, suppose that $u_{j}=P f_{j}$ is the Poisson extension of $f_{j}$. Then, we control $\left|\nabla u_{j}\right|$.

For $u_{1}$, we have $\nabla u_{1}=0$ since $u_{1}$ is a constant.

For $u_{2}$, we ultilize the Littlewood-Paley estimate for $L^{p}$ to derive

$$
\begin{aligned}
& \int_{I}\left(\int_{1-|I|}^{1}\left|\nabla u_{2}(r \zeta)\right|^{2}\left(1-r^{2}\right) \mathrm{d} r\right)^{\frac{p}{2}}|\mathrm{~d} \zeta| \\
& \lesssim \int_{\mathbb{T}}\left(\int_{0}^{1}\left|\nabla u_{2}(r \zeta)\right|^{2}\left(1-r^{2}\right) \mathrm{d} r\right)^{\frac{p}{2}}|\mathrm{~d} \zeta| \\
& \lesssim\left\|f_{2}\right\|_{p}^{p} \\
& \lesssim\|f\|_{p, \eta}^{p}|I|^{\eta}
\end{aligned}
$$

For $u_{3}$, we have that if $z=r e^{i \theta}$ then

$$
\begin{aligned}
& \left|\nabla u_{3}(z)\right| \approx\left|\int_{\mathbb{T}} f_{3}(\zeta)\left(\nabla \frac{1-|z|^{2}}{|\zeta-z|^{2}}\right)\right| \mathrm{d} \zeta|| \\
& \lesssim \int_{0}^{2 \pi} \frac{\left|f_{3}\left(e^{i t}\right)\right|}{(1-r)^{2}+(\theta-t)^{2}} \mathrm{~d} t \\
& \approx \int_{\mathbb{T} \backslash 2 I} \frac{\left|f\left(e^{i t}\right)-f_{I}\right|}{(1-r)^{2}+(\theta-t)^{2}} \mathrm{~d} t \\
& \lesssim \int_{|\theta-t|>|I|} \frac{\left|f\left(e^{i t}\right)-f_{I}\right|}{(\theta-t)^{2}} \mathrm{~d} t \\
& \lesssim \sum_{j \geq 0} \int_{2^{j}|I|<|\theta-t| \leq 2^{j+1}|I|} \frac{\left|f\left(e^{i t}\right)-f_{I}\right|}{\left(2^{j}|I|\right)^{2}} \mathrm{~d} t \\
& \lesssim \sum_{j \geq 0} \frac{\int_{|\theta-t| \leq 2^{j+1}|I|}\left|f\left(e^{i t}\right)-f_{2^{j+1} I}\right| \mathrm{d} t}{\left(2^{j}|I|\right)^{2}}+\sum_{j \geq 0} \frac{\left|f_{2^{j+1} I}-f_{I}\right|}{2^{j}|I|} \\
& \lesssim \sum_{j \geq 0} \frac{1}{\left(2^{j}|I|\right)^{2}}\left(\int_{|\theta-t| \leq 2^{j+1}|I|}\left|f\left(e^{i t}\right)-f_{2^{j+1} I}\right|^{p} \mathrm{~d} t\right)^{\frac{1}{p}}\left(2^{j+1}|I|\right)^{1-\frac{1}{p}} \\
& +\sum_{j \geq 0} \frac{1}{2^{j}|I|}\left(\frac{1}{|I|} \int_{2^{j+1} I}\left|f\left(e^{i t}\right)-f_{2^{j+1} I}\right|^{p} \mathrm{~d} t\right)^{\frac{1}{p}} \\
& \lesssim\|f\|_{p, \eta} \sum_{j \geq 0}\left(2^{j}|I|\right)^{\frac{\eta-1}{p}-1} \\
& \lesssim\|f\|_{p, \eta}|I|^{\frac{\eta-1}{p}-1} \cdot
\end{aligned}
$$


Clearly, the Hölder's inequality has been used in the above estimation. As a further result, we get

$$
\begin{aligned}
& \int_{I}\left(\int_{1-|I|}^{1}\left|\nabla u_{3}(r \zeta)\right|^{2}\left(1-r^{2}\right) \mathrm{d} r\right)^{\frac{p}{2}}|\mathrm{~d} \zeta| \\
& \lesssim|I|^{\left(\frac{\eta-1}{p}-1\right) p}\|f\|_{p, \eta}^{p} \int_{I}\left(\int_{1-|I|}^{1}\left(1-r^{2}\right) \mathrm{d} r\right)^{\frac{p}{2}}|\mathrm{~d} \zeta| \\
& \lesssim\|f\|_{p, \eta}^{p}|I|^{\eta} .
\end{aligned}
$$

Putting the previous estimates for $\left|\nabla u_{j}\right|$ together, we obtain

$$
\|f\|_{p, \eta, \star} \lesssim\|f\|_{p, \eta}<\infty
$$

Conversely, suppose $\|f\|_{p, \eta, \star}<\infty$. To prove $f \in \mathcal{L}_{p, \eta}$, we are required to consider the three cases:

$$
\left\{\begin{array}{l}
0<\eta<1 \\
\eta=1 \\
1<\eta<1+p
\end{array}\right.
$$

The first and second cases are based on a dual-related formula below:

$$
\int_{\mathbb{T}} f(\zeta) \overline{g(\zeta)} \frac{|\mathrm{d} \zeta|}{2 \pi}=\operatorname{Pf}(0) \overline{P g(0)}+\int_{\mathbb{D}} \nabla P f(z) \cdot \overline{\nabla P g(z)} \log \frac{1}{|z|} \mathrm{d} A(z) .
$$

However, the third case follows from a mean value estimate for the harmonic functions.

Case 1: $0<\eta<1$. For simplicity, set $P f=F$ and $P g=G$. It suffices to handle the convergence of the pair:

$$
\langle F, G\rangle:=\int_{\mathbb{D}} \nabla F(z) \cdot \overline{\nabla G(z)}\left(1-|z|^{2}\right) \mathrm{d} A(z) \quad \forall \quad f \in \mathcal{L}_{p, \eta} \&\left(p^{\prime}, \eta\right)-\text { atom } g .
$$

To do so, thanks to Lemma 4 (i) we may assume that $f \in \mathcal{L}_{p, \eta}$ and $g$ is a $\left(p^{\prime}, \eta\right)$-atom generated by a subarc $I \subseteq \mathbb{T}$ with $|I| \ll 1$. Then, we estimate

$$
|\langle F, G\rangle| \lesssim \int_{\mathbb{T}} \int_{0}^{1}|\nabla F(r \zeta)||\nabla G(r \zeta)|(1-r) \mathrm{d} r|\mathrm{~d} \zeta| \approx \iint_{S(2 I)}+\iint_{\mathbb{D} \backslash S(2 I)}
$$

where

$$
\left\{\begin{array}{l}
S(2 I)=\{z=r \zeta: r \in[1-2|I|, 1) \& \zeta \in 2 I\} \\
\iint_{S(2 I)}=\int_{2 I} \int_{1-2|I|}^{1}|\nabla F(r \zeta)||\nabla G(r \zeta)|(1-r) \mathrm{d} r|\mathrm{~d} \zeta| \\
\iint_{\mathbb{D} \backslash S(2 I)}=\iint_{\mathbb{D} \backslash S(2 I)}|\nabla F(r \zeta)||\nabla G(r \zeta)|(1-r) \mathrm{d} r|\mathrm{~d} \zeta|
\end{array}\right.
$$


For $\iint_{S(2 I)}$, we use the Hölder's inequality twice and the $L^{p^{\prime}}$-bound of the LittlewoodPaley $G$-function to obtain

$$
\begin{aligned}
& \iint_{S(2 I)} \lesssim \int_{2 I} \frac{\left(\int_{1-2|I|}^{1}|\nabla F(r \zeta)|^{2}(1-r) \mathrm{d} r\right)^{\frac{1}{2}}}{\left(\int_{1-2|I|}^{1}|\nabla G(r \zeta)|^{2}(1-r) \mathrm{d} r\right)^{-\frac{1}{2}}}|\mathrm{~d} \zeta| \\
& \lesssim\left(\int_{2 I}\left(\int_{1-2|I|}^{1}|\nabla F(r \zeta)|^{2}(1-r) \mathrm{d} r\right)^{\frac{p}{2}}|\mathrm{~d} \zeta|\right)^{\frac{1}{p}} \\
& \times\left(\int_{2 I}\left(\int_{1-2|I|}^{1}|\nabla G(r \zeta)|^{2}(1-r) \mathrm{d} r\right)^{\frac{p^{\prime}}{2}}|\mathrm{~d} \zeta|\right)^{\frac{1}{p^{\prime}}} \\
& \lesssim\left(\int_{2 I}\left(\int_{1-2|I|}^{1}|\nabla F(r \zeta)|^{2}(1-r) \mathrm{d} r\right)^{\frac{p}{2}}|\mathrm{~d} \zeta|\right)^{\frac{1}{p}}\|g\|_{L^{p^{\prime}}} \\
& \lesssim\|f\|_{p, \eta, \star}|I|^{\frac{\eta}{p}}\|g\|_{L^{p^{\prime}}} \\
& \lesssim\|f\|_{p, \eta, \star}
\end{aligned}
$$

For $\iint_{\mathbb{D} \backslash S(2 I)}$, recalling that $g$ is a $\left(p^{\prime}, \eta\right)$-atom associated to $I$ centered at $\zeta_{0}=e^{i \theta_{0}}$, we get

$$
G(z)=\int_{\mathbb{T}} g(\zeta)\left(\frac{1-|z|^{2}}{|\zeta-z|^{2}}-\frac{1-|z|^{2}}{\left|\zeta_{0}-z\right|^{2}}\right) \frac{|\mathrm{d} \zeta|}{2 \pi},
$$

whence deriving

$$
\begin{aligned}
& \nabla G(z)=\int_{\mathbb{T}} g(\zeta) \nabla\left(\frac{1-|z|^{2}}{|\zeta-z|^{2}}-\frac{1-|z|^{2}}{\left|\zeta_{0}-z\right|^{2}}\right) \frac{|\mathrm{d} \zeta|}{2 \pi} \\
& =\int_{I} g(\zeta) \nabla\left(\frac{1-|z|^{2}}{|\zeta-z|^{2}}-\frac{1-|z|^{2}}{\left|\zeta_{0}-z\right|^{2}}\right) \frac{|\mathrm{d} \zeta|}{2 \pi} .
\end{aligned}
$$

To proceed, let

$$
\left[\zeta_{0}, \zeta\right]:=\left\{\phi(t):=\zeta_{0}+\left(\zeta-\zeta_{0}\right) t \in \mathbb{D}: t \in[0,1]\right\}
$$

be the line segment connecting $\zeta \in I$ and $\zeta_{0} \in I$. Since $|\zeta|=\left|\zeta_{0}\right|=1$, we can use the mean value theorem for derivatives to obtain some $t_{0} \in[0,1]$ such that

$$
\begin{aligned}
& \left|\partial_{z}\left(\frac{1-|z|^{2}}{|\zeta-z|^{2}}-\frac{1-|z|^{2}}{\left|\zeta_{0}-z\right|^{2}}\right)\right| \\
& =\left|\partial_{z}\left(\frac{1-|z|^{2}}{|\zeta-z|^{2}}\right)-\partial_{z}\left(\frac{1-|z|^{2}}{\left|\zeta_{0}-z\right|^{2}}\right)\right| \\
& =\left|\partial_{t}\left(\partial_{z}\left(\frac{1-|z|^{2}}{|\phi(t)-z|^{2}}\right)\right)\right|_{t=t_{0}}\left|\zeta-\zeta_{0}\right| \\
& =\left|\partial_{z}\left(\partial_{t}\left(\frac{1-|z|^{2}}{|\phi(t)-z|^{2}}\right)\right)\right|_{t=t_{0}}\left|\zeta-\zeta_{0}\right| .
\end{aligned}
$$


A direct calculation gives

$$
\partial_{t}\left(\frac{1-|z|^{2}}{|\phi(t)-z|^{2}}\right)=2 \Re \phi^{\prime}(t) \overline{(\phi(t)-z)} \frac{1-|z|^{2}}{|\phi(t)-z|^{4}} .
$$

Thus

$$
\begin{aligned}
& \left|\partial_{z}\left(\frac{1-|z|^{2}}{|\zeta-z|^{2}}-\frac{1-|z|^{2}}{\left|\zeta_{0}-z\right|^{2}}\right)\right| \\
& =\left|\partial_{z}\left(2 \Re \phi^{\prime}(t) \overline{(\phi(t)-z)} \frac{1-|z|^{2}}{|\phi(t)-z|^{4}}\right)\right|_{t=t_{0}}\left|\zeta-\zeta_{0}\right| .
\end{aligned}
$$

Noticing

$$
\left\{\begin{array}{l}
\phi^{\prime}(t)=\zeta-\zeta_{0} ; \\
2 \Re \phi^{\prime}(t) \overline{(\phi(t)-z)}=\phi^{\prime}(t) \overline{(\phi(t)-z)}+\overline{\phi^{\prime}(t)}(\phi(t)-z),
\end{array}\right.
$$

we compute

$$
\begin{aligned}
& \partial_{z}\left(\phi^{\prime}(t)(\overline{\phi(t)}-\bar{z}) \frac{1-|z|^{2}}{|\phi(t)-z|^{4}}\right) \\
& =\frac{\phi^{\prime}(t)(\overline{\phi(t)}-\bar{z})}{|\phi(t)-z|^{6}}\left(-\bar{z}|\phi(t)-z|^{2}+2(\overline{\phi(t)}-\bar{z})\right)
\end{aligned}
$$

and

$$
\begin{aligned}
& \partial_{z}\left(\overline{\phi^{\prime}(t)}(\phi(t)-z) \frac{1-|z|^{2}}{|\phi(t)-z|^{4}}\right) \\
& =\frac{\overline{\phi^{\prime}(t)}(\phi(t)-z)}{|\phi(t)-z|^{6}}\left(-\bar{z}|\phi(t)-z|^{2}+2(\overline{\phi(t)}-\bar{z})\right)-\frac{\overline{\phi^{\prime}(t)}\left(1-|z|^{2}\right)}{|\phi(t)-z|^{4}}
\end{aligned}
$$

whence getting

$$
\left|\partial_{z}\left(\partial_{t}\left(\frac{1-|z|^{2}}{|\phi(t)-z|^{2}}\right)\right)\right| \lesssim \frac{\left|\phi^{\prime}(t)\right|}{|\phi(t)-z|^{4}}
$$

If $z \in S\left(2^{j+1} I\right) \backslash S\left(2^{j} I\right)$ (where $j=1,2,3, \ldots$ ) and $t \in[0,1]$, then

$$
|\phi(t)-z| \gtrsim 2^{j}|I| \gtrsim 2^{j}\left|\zeta-\zeta_{0}\right|
$$

and hence

$$
\left|\partial_{z}\left(\frac{1-|z|^{2}}{|\zeta-z|^{2}}-\frac{1-|z|^{2}}{\left|\zeta_{0}-z\right|^{2}}\right)\right| \lesssim \frac{\left|\zeta-\zeta_{0}\right|^{2}}{|\phi(t)-z|^{4}} \lesssim \frac{1}{2^{4 j}|I|^{2}}
$$

Similarly, we have

$$
\left|\partial_{\bar{z}}\left(\frac{1-|z|^{2}}{|\zeta-z|^{2}}-\frac{1-|z|^{2}}{\left|\zeta_{0}-z\right|^{2}}\right)\right| \lesssim \frac{1}{2^{4 j}|I|^{2}}
$$


Hence

$$
\begin{aligned}
& \left|\nabla\left(\frac{1-|z|^{2}}{|\zeta-z|^{2}}-\frac{1-|z|^{2}}{\left|\zeta_{0}-z\right|^{2}}\right)\right| \\
& \lesssim\left|\partial_{z}\left(\frac{1-|z|^{2}}{|\zeta-z|^{2}}-\frac{1-|z|^{2}}{\left|\zeta_{0}-z\right|^{2}}\right)\right|+\left|\partial_{\bar{z}}\left(\frac{1-|z|^{2}}{|\zeta-z|^{2}}-\frac{1-|z|^{2}}{\left|\zeta_{0}-z\right|^{2}}\right)\right| \\
& \lesssim \frac{1}{2^{4 j}|I|^{2}}
\end{aligned}
$$

Therefore, we employ $\|g\|_{p^{\prime}} \lesssim|I|^{-\frac{\eta}{p}}$ and Hölder's inequality to obtain

$$
|\nabla G(z)| \lesssim \frac{\int_{I}|g(\zeta)||\mathrm{d} \zeta|}{2^{4 j}|I|^{2}} \lesssim \frac{|I|^{\frac{1-\eta}{p}-2}}{2^{4 j}} \quad \forall \quad z \in S\left(2^{j+1} I\right) \backslash S\left(2^{j} I\right)
$$

\section{Upon writing}

$$
\iint_{\mathbb{D} \backslash S(2 I)}=\sum_{j \geq 1} \int_{2^{j} I} \int_{1-2^{j+1}|I|}^{1-2^{j}|I|}+\sum_{j \geq 1} \int_{2^{j+1} \backslash 2^{j} I} \int_{1-2^{j+1}|I|}^{1},
$$


we have two types of estimates as follows. The first type is:

$$
\begin{aligned}
& \sum_{j \geq 1} \int_{2^{j} I} \int_{1-2^{j+1}|I|}^{1-2^{j}|I|} \\
& \lesssim \sum_{j \geq 1} \int_{2^{j} I}\left(\left(\int_{1-2^{j+1}|I|}^{1-2^{j}|I|}|\nabla F(r \zeta)|^{2}(1-r) \mathrm{d} r\right)^{\frac{1}{2}}\right. \\
& \left.\times\left(\int_{1-2^{j+1}|I|}^{1-2^{j}|I|}|\nabla G(r \zeta)|^{2}(1-r) \mathrm{d} r\right)^{\frac{1}{2}}\right)|\mathrm{d} \zeta| \\
& \lesssim \sum_{j \geq 1}\left(\int_{2^{j} I}\left(\int_{1-2^{j+1}|I|}^{1-2^{j}|I|}|\nabla F(r \zeta)|^{2}(1-r) \mathrm{d} r\right)^{\frac{p}{2}}|\mathrm{~d} \zeta|\right)^{\frac{1}{p}} \\
& \times\left(\int_{2^{j} I}\left(\int_{1-2^{j+1}|I|}^{1-2^{j}|I|}|\nabla G(r \zeta)|^{2}(1-r) \mathrm{d} r\right)^{\frac{p^{\prime}}{2}}|\mathrm{~d} \zeta|\right)^{\frac{1}{p^{\prime}}} \\
& \lesssim\|f\|_{p, \eta, \star} \sum_{j \geq 1}\left|2^{j} I\right|^{\eta / p}\left(\int_{2^{j} I}\left(\int_{1-2^{j+1}|I|}^{1-2^{j}|I|}\left(\frac{|I|^{\frac{1-\eta}{p}-2}}{2^{4 j}}\right)^{2}(1-r) \mathrm{d} r\right)^{\frac{p^{\prime}}{2}}|\mathrm{~d} \zeta|\right)^{\frac{1}{p^{\prime}}} \\
& \lesssim\|f\|_{p, \eta, \star}|I|^{\frac{\eta}{p}+\left(\frac{1-\eta}{p}-2\right)} \sum_{j \geq 1} 2^{(j+1) \frac{\eta}{p}-4 j}\left(\int_{2^{j} I}\left(\int_{1-2^{j+1}|I|}^{1}(1-r) \mathrm{d} r\right)^{\frac{p^{\prime}}{2}}|\mathrm{~d} \zeta|\right)^{\frac{1}{p^{\prime}}} \\
& \lesssim\|f\|_{p, \eta, \star}|I|^{\frac{1}{p}-2} \sum_{j \geq 1} 2^{(j+1) \frac{\eta}{p}-4 j}\left(2^{j+1}|I|\left(2^{j+1}|I|\right)^{p^{\prime}}\right)^{\frac{1}{p^{\prime}}} \\
& \lesssim\|f\|_{p, \eta, \star}|I|^{\frac{1}{p}-2+1+\frac{1}{p^{\prime}}} \sum_{j \geq 1} 2^{(j+1) \frac{\eta}{p}-4 j+j+1+\frac{j+1}{p^{\prime}}} \\
& \lesssim\|f\|_{p, \eta, \star} \sum_{j \geq 1} 2^{j\left(\frac{\eta-1}{p}-2\right)} \\
& \lesssim\|f\|_{p, \eta, \star} .
\end{aligned}
$$

In the above we have used the Hölder's inequality twice, the estimate for $|\nabla G|$ on $S\left(2^{j+1} I\right) \backslash S\left(2^{j} I\right)$ and the assumption $0<\eta<1$.

The second type is:

$$
\sum_{j \geq 1} \int_{2^{j+1} \backslash 2^{j} I} \int_{1-2^{j+1}|I|}^{1} \lesssim\|f\|_{p, \eta, \star} \sum_{j \geq 1} 2^{j\left(\frac{\eta-1}{p}-2\right)},
$$

which may be verfied in a way similar to the first type. As a result, we have

$$
\iint_{\mathbb{D} \backslash S(2 I)} \lesssim\|f\|_{p, \eta, \star}
$$

whence reaching

$$
|\langle F, G\rangle| \lesssim\|f\|_{p, \eta, \star}
$$


This in turn implies $f \in \mathcal{L}_{p, \eta}$.

Case 2: $\eta=1$. Regarding this situation, we apply $(\infty, 1)$-atoms (due to Lemma 4 (ii)) to the previous discussion, and then obtain

$$
|\langle F, G\rangle| \lesssim\|f\|_{p, 1, \star}
$$

which yields $f \in B M O$.

Case 3: $1<\eta<1+p$. Suppose $\|f\|_{p, \eta, \star}<\infty$ and set $u=P f$. For a given $z=r e^{i \theta} \in \mathbb{D}$, let $S(I)$ be the Carleson box with $|I|=(1-|z|)$ and $e^{i \theta}$ be the center of I. Then

$$
|I|^{\eta}\|f\|_{p, \eta, \star}^{p} \gtrsim \int_{I}\left(\int_{1-|I|}^{1}\left|\nabla u\left(r e^{i \theta}\right)\right|^{2}(1-r) \mathrm{d} r\right)^{\frac{p}{2}} \mathrm{~d} \theta .
$$

Since $u$ is harmonic in $\mathbb{D}, \nabla u$ is harmonic overthere as well. Consequently, there exists some number $\rho>0$ such that $B\left(z, \frac{1-|z|}{\rho}\right) \subset S(I)$, where $B\left(z, \frac{1-|z|}{\rho}\right)$ is the open disk with center $z$ and radius $\frac{1-|z|}{\rho}$. This implies the following mean value inequality:

$$
(1-|z|)|\nabla u(z)| \lesssim(1-|z|)^{-2} \int_{B\left(z, \frac{1-|z|}{\rho}\right)}|\nabla u(w)|(1-|w|) \mathrm{d} A(w) .
$$

So, it follows from the Hölder's inequality that

$$
\begin{aligned}
& (1-|z|)|\nabla u(z)| \\
& \lesssim(1-|z|)^{-2} \int_{S(I)}|\nabla u(w)|(1-|w|) \mathrm{d} A(w) \\
& \lesssim(1-|z|)^{-2} \int_{I}\left(\int_{1-|I|}^{1}\left|\nabla u\left(r e^{i \theta}\right)\right|^{2}(1-|r|) \mathrm{d} r\right)^{\frac{1}{2}}\left(\int_{1-|I|}^{1}(1-r) \mathrm{d} r\right)^{\frac{1}{2}} \mathrm{~d} \theta \\
& \lesssim(1-|z|)^{-1} \int_{I}\left(\int_{1-|I|}^{1}\left|\nabla u\left(r e^{i \theta}\right)\right|^{2}(1-|r|) \mathrm{d} r\right)^{\frac{1}{2}} \mathrm{~d} \theta \\
& \lesssim \frac{1}{|I|}\left(\int_{I}\left(\int_{1-|I|}^{1}\left|\nabla u\left(r e^{i \theta}\right)\right|^{2}(1-|r|) \mathrm{d} r\right)^{\frac{p}{2}} \mathrm{~d} \theta\right)^{\frac{1}{p}}|I|^{1-\frac{1}{p}} \\
& \lesssim|I|^{\frac{\eta-1}{p}}\|f\|_{p, \eta, \star} \\
& \lesssim(1-|z|)^{\frac{\eta-1}{p}}\|f\|_{p, \eta, \star \cdot}
\end{aligned}
$$

This means that

$$
(1-|z|)^{\frac{1-\eta}{p}+1}|\nabla u(z)| \lesssim\|f\|_{p, \eta, \star}
$$

and thus

$$
f \in \operatorname{Lip}_{\frac{\eta-1}{p}}=\mathcal{L}_{p, \eta} \quad \text { under } \quad 1<\eta<1+p
$$




\section{Analytic Campanato spaces in Bloch type spaces}

3.1. Generalized Carleson measures. For $\eta>0$, we write $\mathcal{C} \mathcal{M}_{\eta}$ for the class of all $\eta$-Carleson measures on $\mathbb{D}$. Recall that a nonnegative Borel measure $\mu$ on $\mathbb{D}$ is called an $\eta$-Carleson measure provided

$$
\|\mu\|_{\mathcal{C M}_{\eta}}=\sup _{I \subseteq \mathbb{T}} \frac{\mu(S(I))}{|I|^{\eta}}<\infty
$$

where

$$
S(I)=\left\{z=r e^{i \theta} \in \mathbb{D}: 1-|I| \leq r<1 \& e^{i \theta} \in I\right\}
$$

is the Carleson square based on a subarc $I \subseteq \mathbb{T}$.

For $a, b>0$ we define an integral operator $T_{a, b}$ as

$$
T_{a, b} f(z)=\int_{\mathbb{D}} \frac{\left(1-|w|^{2}\right)^{b-1}}{|1-\bar{w} z|^{a+b}} f(w) \mathrm{d} A(w) \quad \forall \quad z \in \mathbb{D} .
$$

Below is a re-statement of Lemma 3.1.2 in [27].

Lemma 6. Let $\eta \in(0,2), a>\frac{2-\eta}{2}, b>\frac{1+\eta}{2}$ and $f$ be Lebesgue measurable on $\mathbb{D}$. If $|f(z)|^{2}\left(1-|z|^{2}\right)^{\eta} \mathrm{d} A(z)$ belongs to $\mathcal{C M}_{\eta}$, then $\left|T_{a, b} f(z)\right|^{2}\left(1-|z|^{2}\right)^{\eta+2 a-2} \mathrm{~d} A(z)$ also belongs to $\mathcal{C M}_{\eta}$.

3.2. Distance estimates. Importantly, Theorem [5](ii) and the mean value inequality for the subharmonic functions (see also the treatment for Case 3: $1<\eta<1+p$ in the proof of Theorem 5) are employed to derive the following optimal inclusion:

$$
0<\eta \leq 1+p \& 1 \leq p<\infty \Longrightarrow \mathcal{A L}_{p, \eta} \subseteq \mathcal{B}_{(p+1-\eta) / p} .
$$

This embedding, along with the P. Jone's distance estimation from the Bloch functions to $B M O A$ (cf. [9, 29, 27]), suggests us to consider the distance of an $\mathcal{B}_{(p+1-\eta) / p}$ function $f$ to $\mathcal{A} \mathcal{L}_{p, \eta}$. Such a distance is defined by

$$
\operatorname{dist}_{\mathcal{B}_{(p+1-\eta) / p}}\left(f, \mathcal{A L}_{p, \eta}\right)=\inf _{g \in \mathcal{A} \mathcal{L}_{p, \eta}}\|f-g\|_{\mathcal{B}_{(p+1-\eta) / p}} \quad \forall \quad f \in \mathcal{B}_{(p+1-\eta) / p} .
$$

Motivated by Theorem 3.1.3 in [27], we get the following characterization of the above-defined distance, approaching the second part of Theorem 1(ii).

Theorem 7. For $\varepsilon>0, \eta \in(0,2)$ and $f \in \mathcal{B}_{(3-\eta) / 2}$, let

$$
\Omega_{\varepsilon}(f)=\left\{z \in \mathbb{D}:\left(1-|z|^{2}\right)^{(3-\eta) / 2}\left|f^{\prime}(z)\right| \geq \varepsilon\right\} .
$$

Then

$$
\operatorname{dist}_{\mathcal{B}_{(3-\eta) / 2}}\left(f, \mathcal{A L}_{2, \eta}\right)=\inf \left\{\varepsilon>0: \frac{\chi_{\Omega_{\varepsilon}(f)}(z)}{\left(1-|z|^{2}\right)^{2-\eta}} \mathrm{d} A(z) \text { is in } \mathcal{C M}_{\eta}\right\} .
$$

Proof. For $f \in \mathcal{B}_{(3-\eta) / 2}$ and $z \in \mathbb{D}$, one has the following representation formula (see, for example, [1, (1.1)] or [27, p. 55]):

$$
f(z)=f(0)+C \int_{\mathbb{D}} \frac{\left(1-|w|^{2}\right)^{(3-\eta) / 2} f^{\prime}(w)}{\bar{w}(1-\bar{w} z)^{1+(3-\eta) / 2}} \mathrm{~d} A(w)=: f_{1}(z)+f_{2}(z),
$$


where

$$
\left\{\begin{array}{l}
f_{1}(z)=f(0)+C \int_{\Omega_{\varepsilon}(f)} \frac{\left(1-|w|^{2}\right)^{(3-\eta) / 2} f^{\prime}(w)}{\bar{w}(1-\bar{w} z)^{1+(3-\eta) / 2}} \mathrm{~d} A(w) ; \\
f_{2}(z)=C \int_{\mathbb{D} \backslash \Omega_{\varepsilon}(f)} \frac{\left(1-|w|^{2}\right)^{(3-\eta) / 2} f^{\prime}(w)}{\bar{w}(1-\bar{w} z)^{1+(3-\eta) / 2}} \mathrm{~d} A(w) ;
\end{array}\right.
$$

with $C$ being a constant depending only on $\eta$. Then

$$
\begin{aligned}
& \left|f_{1}^{\prime}(z)\right| \lesssim\|f\|_{\mathcal{B}_{(3-\eta) / 2}} \int_{\mathbb{D}} \frac{\chi_{\Omega_{\varepsilon}(f)}(w)}{|1-\bar{w} z|^{2+(3-\eta) / 2}} \mathrm{~d} A(w) \\
& \approx\|f\|_{\mathcal{B}_{(3-\eta) / 2}} \int_{\mathbb{D}} \frac{\left(1-|w|^{2}\right)}{|1-\bar{w} z|^{2+(3-\eta) / 2}}\left(\frac{\chi_{\Omega_{\varepsilon}(f)}(w)}{1-|w|^{2}}\right) \mathrm{d} A(w)
\end{aligned}
$$

So, if

$$
\left(\chi_{\Omega_{\varepsilon}(f)}(z)\right)^{2}\left(1-|z|^{2}\right)^{\eta-2} \mathrm{~d} A(z)
$$

is in $\mathcal{C M}_{\eta}$, Lemma 6 (with $a=(3-\eta) / 2$ and $b=2$ ) implies that

$$
\left|f_{1}^{\prime}(z)\right|^{2}\left(1-|z|^{2}\right) \mathrm{d} A(z)
$$

belongs to $\mathcal{C} \mathcal{M}_{\eta}$. This means $f_{1} \in \mathcal{A} \mathcal{L}_{2, \eta}$. Meanwhile, we have

$$
\left|f_{2}^{\prime}(z)\right| \lesssim \varepsilon \int_{\mathbb{D}}|1-\bar{w} z|^{(\eta-3) / 2-2} \mathrm{~d} A(w) \lesssim \frac{\varepsilon}{\left(1-|w|^{2}\right)^{(3-\eta) / 2}}
$$

This gives

$$
\operatorname{dist}_{\mathcal{B}_{(3-\eta) / 2}}\left(f, \mathcal{A L}_{2, \eta}\right) \leq \inf \left\{\varepsilon>0: \chi_{\Omega_{\varepsilon}(f)}(z)\left(1-|z|^{2}\right)^{\eta-2} \mathrm{~d} A(z) \in \mathcal{C M}_{\eta}\right\} .
$$

In order to prove that the last inequality is actually an equality, we may assume that $\varepsilon_{0}$ equals the right-hand quantity of the last inequality and

$$
\operatorname{dist}_{\mathcal{B}_{(3-\eta) / 2}}\left(f, \mathcal{A L}_{2, \eta}\right)<\varepsilon_{0} .
$$

It is enough to consider the case $\varepsilon_{0}>0$. Under $\varepsilon_{0}>0$ there exists an $\varepsilon_{1}$ such that

$$
0<\varepsilon_{1}<\varepsilon_{0} \& \operatorname{dist}_{\mathcal{B}_{(3-\eta) / 2}}\left(f, \mathcal{A L}_{2, \eta}\right)<\varepsilon_{1} .
$$

Hence, by definition, we can find a function $g \in \mathcal{A L}_{2, \eta}$ such that

$$
\|f-g\|_{\mathcal{B}_{(3-\eta) / 2}}<\varepsilon_{1} .
$$

Now for any $\varepsilon \in\left(\varepsilon_{1}, \varepsilon_{0}\right)$ we have that

$$
\chi_{\Omega_{\varepsilon}(f)}(z)\left(1-|z|^{2}\right)^{\eta-2} \mathrm{~d} A(z)
$$

is not in $\mathcal{C M}_{\eta}$. But, $\|f-g\|_{\mathcal{B}_{(3-\eta) / 2}}<\varepsilon_{1}$ yields

$$
\left(1-|z|^{2}\right)^{(3-\eta) / 2}\left|g^{\prime}(z)\right|>\left(1-|z|^{2}\right)^{(3-\eta) / 2}\left|f^{\prime}(z)\right|-\varepsilon_{1} \quad \forall z \in \mathbb{D},
$$

and so

$$
\chi_{\Omega_{\varepsilon}(f)}(z) \leq \chi_{\Omega_{\varepsilon-\varepsilon_{1}}(g)}(z) \quad \forall \quad z \in \mathbb{D} .
$$

This implies that

$$
\chi_{\Omega_{\varepsilon-\varepsilon_{1}}(g)}(z)\left(1-|z|^{2}\right)^{\eta-2} \mathrm{~d} A(z)
$$


does not belong to $\mathcal{C M}_{\eta}$. However,

$$
\begin{aligned}
& \chi_{\Omega_{\varepsilon-\varepsilon_{1}}(g)}(z)\left(1-|z|^{2}\right)^{\eta-2} \mathrm{~d} A(z) \\
& \leq \chi_{\Omega_{\varepsilon-\varepsilon_{1}}(g)}(z) \frac{\left(1-|z|^{2}\right)}{\left(1-|z|^{2}\right)^{3-\eta}} \mathrm{d} A(z) \\
& \leq \frac{\left|g^{\prime}(z)\right|^{2}}{\left(\varepsilon-\varepsilon_{1}\right)^{2}}\left(1-|z|^{2}\right) \chi_{\Omega_{\varepsilon-\varepsilon_{1}}(g)}(z) \mathrm{d} A(z) \\
& \leq\left(\varepsilon-\varepsilon_{1}\right)^{-2}\left|g^{\prime}(z)\right|^{2}\left(1-|z|^{2}\right) \mathrm{d} A(z) .
\end{aligned}
$$

Since $g \in \mathcal{A L}_{2, \eta}$ means that

$$
\left|g^{\prime}(z)\right|^{2}\left(1-|z|^{2}\right) \mathrm{d} A(z)
$$

is in $\mathcal{C M}_{\eta}$, and consequently

$$
\chi_{\Omega_{\varepsilon-\varepsilon_{1}}(g)}(z)\left(1-|z|^{2}\right)^{\eta-2} \mathrm{~d} A(z)
$$

is in $\mathcal{C M}_{\eta}$. Now, a contradiction occurs. Thus we must have

$$
\operatorname{dist}_{\mathcal{B}_{(3-\eta) / 2}}\left(f, \mathcal{A} \mathcal{L}_{2, \eta}\right)=\varepsilon_{0}
$$

as required.

\section{Remark 8.}

(i) Theorem 7 characterizes the closure of $\mathcal{A L}_{2, \eta}$ in the $\mathcal{B}_{(3-\eta) / 2}$ norm. That is, for $f \in \mathcal{B}_{(3-\eta) / 2}$, $f$ is in the closure of $\mathcal{A L}_{2, \eta}$ in the $\mathcal{B}_{(3-\eta) / 2}$ norm if and only if for every $\varepsilon>0$

$$
\int_{\Omega_{\varepsilon}(f) \cap S(I)}\left(1-|z|^{2}\right)^{\eta-2} \mathrm{~d} A(z) \lesssim|I|^{\eta}
$$

holds for any Carleson square $S(I) \subseteq \mathbb{D}$.

(ii) The proof of Theorem 7 depends on an important fact that $f \in \mathcal{A} \mathcal{L}_{2, \eta}$ if and only if $\left|f^{\prime}(z)\right|^{2}\left(1-|z|^{2}\right) \mathrm{d} A(z)$ is a member of $\mathcal{C M}_{\eta}$. Given $1<p<\infty$, if we define the analytic function space $\mathcal{C} \mathcal{A}_{p, \eta}$ to be the space of all $H^{p}$ functions satisfying

$$
\|f\|_{\mathcal{C A}_{p, \eta}}^{p}=\left\|\left|f^{\prime}(z)\right|^{p}\left(1-|z|^{2}\right)^{p-1} \mathrm{~d} A(z)\right\|_{\mathcal{C M}_{\eta}}<\infty
$$

then $\mathcal{C A}_{2, \eta}=\mathcal{A} \mathcal{L}_{2, \eta}$. This $\mathcal{C} \mathcal{A}_{p, \eta}$ is closely related to $\mathcal{A L}_{p, \eta}$. It can also be proved that $\mathcal{C} \mathcal{A}_{p, \eta} \subseteq \mathcal{B}_{1+(1-\eta) / p}$. In a future article, we will characterize the distance of a function in $\mathcal{B}_{1+(1-\eta) / p}$ to $\mathcal{C} \mathcal{A}_{p, \eta}$ under $0<\eta \leq 1+p$ and $1 \leq p<\infty$.

\section{Compositions For anAlytic CAMPANATO SPACES}

4.1. Actions between analytic Campanato spaces. In what follows, for $0<r<1$ and an analytic self-map $\varphi$ of $\mathbb{D}$ let

$$
N_{r}(\varphi, w)=\sum_{z \in \varphi^{-1}\{w\}} \log ^{+}\left(\frac{r}{|z|}\right) \quad \forall \quad w \in \mathbb{D},
$$

where $\log ^{+} x=\max \{\log x, 0\}$, and then set

$$
N(\varphi, w)=\lim _{r \rightarrow 1} N_{r}(\varphi, w) \quad \forall \quad w \in \mathbb{D}
$$


be the Nevanlinna counting function of $\phi$. The importance of such a counting function in the study of compositions on $H^{p}$ (cf. [5, 11, 19, 20]) initially comes from the following fundamental formula (cf. [21, p. 2336]).

Lemma 9. Let $\varphi$ be an analytic self-map of $\mathbb{D}$ and $f \in H^{p}$ with $p \in[1, \infty)$. Then

$$
\|f \circ \varphi\|_{p}^{p}=|f(\varphi(0))|^{p}+\frac{p^{2}}{2} \int_{\mathbb{D}}|f(w)|^{p-2}\left|f^{\prime}(w)\right|^{2} N(\varphi, w) \mathrm{d} A(w) .
$$

In particular,

$$
\|f\|_{p}^{p}=|f(0)|^{p}+\frac{p^{2}}{2} \int_{\mathbb{D}}|f(w)|^{p-2}\left|f^{\prime}(w)\right|^{2} \log \frac{1}{|w|} \mathrm{d} A(w) .
$$

This is used to establish two precise estimates for $\|\varphi\|_{p}$ extending [13, (2.8)].

Lemma 10. If $\varphi$ is an analytic self-map of $\mathbb{D}$ with $\varphi(0)=0$, then

(i)

(ii)

$$
\|\varphi\|_{2}^{2}=2 \int_{\mathbb{D}} N(\varphi, w) \mathrm{d} A(w)
$$

$$
N(\varphi, z) \leq \frac{4}{\log 2}\|\varphi\|_{2}^{2} \log \frac{1}{|z|} \quad \forall \quad z \in \mathbb{D} \backslash \frac{1}{2} \mathbb{D} .
$$

Proof. (i) This follows from taking $f(z)=z$ in Lemma 9 .

(ii) Given $w \in \mathbb{D} \backslash\{0\}$. Using (i) and the submean value property of $N(\varphi, \cdot)$, we get

$$
N(\varphi, w) \leq \frac{1}{(1-|w|)^{2}} \int_{|z-w|<1-|w|} N(\varphi, z) \mathrm{d} A(z) \leq \frac{\|\varphi\|_{p}^{p}}{(1-|w|)^{2}} .
$$

Consequently,

$$
N_{r}(\varphi, w) \leq N(\varphi, w) \leq 4\|\varphi\|_{2}^{2} \text { for }|w|=1 / 2 .
$$

Note that the well-known Littlewood's inequality ensures that $N_{r}(\varphi, w)$ approaches zero uniformly as $|w| \rightarrow 1$. Note also that $N_{r}(\varphi, w)$ is subharmonic. So it is bounded above by the harmonic function on the annulus $\{w \in \mathbb{D}: 1 / 2<|w|<1\}$ having these boundary values. Hence,

$$
N(\varphi, w)=\lim _{r \rightarrow 1} N_{r}(\varphi, w) \leq \frac{4}{\log 2}\|\varphi\|_{2}^{2} \log \frac{1}{|w|} \quad \text { for } \quad 1 / 2<|w|<1 .
$$

As the generalization of an $H^{2}$ composition result in [13], the following splitting inequality essentially improves the well-known sub-ordination principle for $H^{p}$ with $p \geq 2$.

Theorem 11. Let $2 \leq p<\infty$. Then

$$
\|f \circ \varphi\|_{p} \lesssim\|f\|_{p}\|\varphi\|_{p}^{2 / p}
$$

holds for all $f \in H^{p}$ and analytic self-maps $\varphi$ of $\mathbb{D}$ with $f(0)=\varphi(0)=0$. 
Proof. According to Lemma 9, we have

$$
\|f \circ \varphi\|_{p}^{p}=\frac{p^{2}}{2} \int_{\mathbb{D}}|f(w)|^{p-2}\left|f^{\prime}(w)\right|^{2} N(\varphi, w) \mathrm{d} A(w)=: \int_{\frac{1}{2} \mathbb{D}}+\int_{\mathbb{D} \backslash \frac{1}{2} \mathbb{D}} .
$$

Using the Cauchy integral formula and Hölder's inequality, we get

$$
|f(z)|+(1-|z|)\left|f^{\prime}(z)\right| \lesssim\|f\|_{p}(1-|z|)^{-\frac{1}{p}} \quad \forall \quad z \in \mathbb{D} .
$$

Thus by Lemma 10 (i) and $p \geq 2$, we find

$$
\begin{aligned}
& \int_{\frac{1}{2} \mathbb{D}}=\frac{p^{2}}{2} \int_{\frac{1}{2} \mathbb{D}}|f(w)|^{p-2}\left|f^{\prime}(w)\right|^{2} N(\varphi, w) \mathrm{d} A(w) \\
& \lesssim\|f\|_{p}^{p} \int_{\frac{1}{2} \mathbb{D}} N(\varphi, w)(1-|w|)^{-p-2} \mathrm{~d} A(w) \\
& \lesssim\|f\|_{p}^{p} \int_{\frac{1}{2} \mathbb{D}} N(\varphi, w) \mathrm{d} A(w) \\
& \lesssim\|\varphi\|_{2}^{2}\|f\|_{p}^{p}
\end{aligned}
$$

Meanwhile, Lemma10(ii) implies

$$
\begin{aligned}
& \int_{\mathbb{D} \backslash \frac{1}{2} \mathbb{D}}=\frac{p^{2}}{2} \int_{\mathbb{D} \backslash \frac{1}{2} \mathbb{D}}|f(w)|^{p-2}\left|f^{\prime}(w)\right|^{2} N(\varphi, w) \mathrm{d} A(w) \\
& \lesssim\|\varphi\|_{2}^{2} \int_{\mathbb{D} \backslash \frac{1}{2} \mathbb{D}}|f(w)|^{p-2}\left|f^{\prime}(w)\right|^{2} \log \frac{1}{|w|} \mathrm{d} A(w) \\
& \lesssim\|\varphi\|_{2}^{2}\|f\|_{p}^{p} .
\end{aligned}
$$

Now, since $p \geq 2$ the above estimates and the Hölder inequality are utilized to derive

$$
\|f \circ \varphi\|_{p}^{p} \lesssim\|\varphi\|_{2}^{2}\|f\|_{p}^{p} \lesssim\|\varphi\|_{p}^{2}\|f\|_{p}^{p}
$$

as desired.

We have the following assertion which covers Theorem 1(iii) and implies that $\mathcal{A} \mathcal{L}_{p, \eta}$ embeds into $\mathcal{A L}_{q, \lambda}$ under $p \geq q=2$ and $(1-\lambda) p \geq(1-\eta) q$.

Theorem 12. Let $0<\eta, \lambda<2=q \leq p<\infty$ and $\varphi$ be an analytic self-map of $\mathbb{D}$. Then

$$
\left\|C_{\varphi} f\right\|_{\mathcal{A} \mathcal{L}_{q, \lambda, *}} \lesssim\|f\|_{\mathcal{A L}_{p, \eta, *}} \quad \forall \quad f \in \mathcal{A} \mathcal{L}_{p, \eta}
$$

when and only when

$$
\sup _{w \in \mathbb{D}} \frac{\left(1-|w|^{2}\right)^{(1-\lambda) / q}}{\left(1-|\varphi(w)|^{2}\right)^{(1-\eta) / p}}\left\|\sigma_{\varphi(w)} \circ \varphi \circ \sigma_{w}\right\|_{q}<\infty .
$$


Proof. If $f \in \mathcal{A L}_{p, \eta}$ and $2=q \leq p$, then an application of Theorem 11, plus Hölder's inequality, derives

$$
\begin{aligned}
& \left\|C_{\varphi}(f)\right\|_{\mathcal{A} \mathcal{L}_{q, \lambda, *}}^{q} \approx \sup _{w \in \mathbb{D}}\left(1-|w|^{2}\right)^{1-\lambda}\left\|f \circ \varphi \circ \sigma_{w}-f(\varphi(w))\right\|_{q}^{q} \\
& \approx \sup _{w \in \mathbb{D}}\left(1-|w|^{2}\right)^{1-\lambda}\left\|\left(f \circ \sigma_{\varphi(w)}-f(\varphi(w))\right) \circ\left(\sigma_{\varphi(w)} \circ \varphi \circ \sigma_{w}\right)\right\|_{q}^{q} \\
& \lesssim \sup _{w \in \mathbb{D}}\left(1-|w|^{2}\right)^{1-\lambda}\left\|f \circ \sigma_{\varphi(w)}-f(\varphi(w))\right\|\left\|_{p}^{q}\right\| \sigma_{\varphi(w)} \circ \varphi \circ \sigma_{w} \|_{q}^{q} \\
& \lesssim \sup _{w \in \mathbb{D}} \frac{\left(1-|w|^{2}\right)^{1-\lambda}}{\left(1-|\varphi(w)|^{2}\right)^{q(1-\eta) / p}}\left\|\sigma_{\varphi(w)} \circ \varphi \circ \sigma_{w}\right\|_{q}^{q}\|f\|_{p, \eta, *}^{q}
\end{aligned}
$$

and hence the forward implication holds.

To verify the backward implication, for $b, z \in \mathbb{D}$ let $f_{b}(z)=\frac{1}{1-b z}$. Then

$$
\begin{aligned}
& \left\|f_{b}\right\|_{\mathcal{A} \mathcal{L}_{p, \eta, *}}^{p} \approx \sup _{w \in \mathbb{D}}\left(1-|w|^{2}\right)^{1-\eta}\left\|f_{b} \circ \sigma_{w}-f_{b}(w)\right\|_{p}^{p} \\
& \approx \sup _{w \in \mathbb{D}}\left(1-|w|^{2}\right)^{1-\eta} \int_{\mathbb{T}}\left|f_{b}(\zeta)-f_{b}(w)\right|^{p} \frac{1-|w|^{2}}{|\zeta-w|^{2}}|\mathrm{~d} \zeta| \\
& \approx \sup _{w \in \mathbb{D}}\left(1-|w|^{2}\right)^{1-\eta} \int_{\mathbb{T}}\left|\frac{\bar{b}(\zeta-w)}{(1-\bar{b} \zeta)(1-\bar{b} w)}\right|^{p} \frac{1-|w|^{2}}{|\zeta-w|^{2}}|\mathrm{~d} \zeta| \\
& \approx \sup _{w \in \mathbb{D}} \frac{|b|^{p}\left(1-|w|^{2}\right)^{1-\eta}}{|1-\bar{b} w|^{p}} \int_{\mathbb{T}}\left|\frac{\zeta-w}{1-\bar{b} \zeta}\right|^{p} \frac{1-|w|^{2}}{|\zeta-w|^{2}}|\mathrm{~d} \zeta| \\
& \approx \sup _{w \in \mathbb{D}} \frac{|b|^{p}\left(1-|w|^{2}\right)^{1-\eta}}{|1-\bar{b} w|^{p}} \int_{\mathbb{T}}\left|\frac{w-\sigma_{w}(\zeta)}{1-\bar{b} \sigma_{w}(\zeta)}\right|^{p}|\mathrm{~d} \zeta|^{2} \\
& \approx \sup _{w \in \mathbb{D}} \frac{|b|^{p}\left(1-|w|^{2}\right)^{1-\eta}}{|1-\bar{b} w|^{p}} \int_{\mathbb{T}} \mid \frac{1-|w|^{2}}{1-\bar{b} w-\bar{w} \zeta+\left.\bar{b} \zeta\right|^{p}|\mathrm{~d} \zeta|} \\
& \approx \sup _{w \in \mathbb{D}} \frac{|b|^{p}\left(1-|w|^{2}\right)^{p+1-\eta}}{|1-\bar{b} w|^{2 p}} \int_{\mathbb{T}}\left|\frac{1}{1-\sigma_{\bar{b}}(\bar{w}) \zeta}\right|^{p}|\mathrm{~d} \zeta|^{p} \\
& \lesssim \sup _{w \in \mathbb{D}} \frac{|b|^{p}\left(1-|w|^{2}\right)^{p+1-\eta}}{|1-\bar{b} w|^{2 p}}\left(1-\left|\sigma_{\bar{b}}(\bar{w})\right|\right)^{1-p} \\
& \approx \sup _{w \in \mathbb{D}} \frac{|b|^{p}\left(1-|w|^{2}\right)^{2-\eta}}{|1-\bar{b} w|^{2}\left(1-|b|^{2}\right)^{p-1}}
\end{aligned}
$$

If

$$
F_{b}(z)=\left(1-|b|^{2}\right)^{\frac{p+\eta-1}{p}} /(1-\bar{b} z) \quad \forall \quad z \in \mathbb{D},
$$

then

$$
\left\|F_{b}\right\|_{\mathcal{A} \mathcal{L}_{p, \eta, *}} \lesssim \sup _{w \in \mathbb{D}} \frac{|b|^{p}\left(1-|w|^{2}\right)^{2-\eta}\left(1-|b|^{2}\right)^{\eta}}{|1-\bar{b} w|^{2}} \lesssim 1,
$$

and hence $F_{b}$ is a bounded function in $\mathcal{A} \mathcal{L}_{p, \eta}$ with a bound being independent of $b$.

Now, let

$$
\left\|C_{\varphi} f\right\|_{\mathcal{A L}_{q, \lambda, *}} \lesssim\|f\|_{\mathcal{A L}_{p, \eta, *}} \quad \forall \quad f \in \mathcal{A L}_{p, \eta} .
$$


Then for $\tau:=(p+\eta-1) / p$ one has

$$
\begin{aligned}
& 1 \gtrsim\left\|F_{b}\right\|_{\mathcal{A} \mathcal{L}_{p, \eta, *}}^{q} \\
& \gtrsim\left\|C_{\varphi} F_{b}\right\|_{\mathcal{A L}_{q, \lambda, *}}^{q} \\
& \approx \sup _{b, w \in \mathbb{D}}\left(1-|w|^{2}\right)^{1-\lambda} \int_{\mathbb{T}}\left|F_{b} \circ \varphi \circ \sigma_{w}-F_{b}(\varphi(w))\right|^{q}|\mathrm{~d} \zeta| \\
& \approx \sup _{b, w \in \mathbb{D}}\left(1-|w|^{2}\right)^{1-\lambda} \int_{\mathbb{T}}\left|F_{b} \circ \varphi-F_{b}(\varphi(w))\right|^{q}\left|\sigma_{w}^{\prime}(\zeta)\right||\mathrm{d} \zeta| \\
& \approx \sup _{b, w \in \mathbb{D}}\left(1-|w|^{2}\right)^{1-\lambda} \int_{\mathbb{T}}\left|\frac{\left(1-|b|^{2}\right)^{\tau}}{(1-\bar{b} \varphi(\zeta))}-\frac{\left(1-|b|^{2}\right)^{\tau}}{(1-\bar{b} \varphi(w))}\right|^{q}\left|\sigma_{w}^{\prime}(\zeta)\right||\mathrm{d} \zeta| \\
& \approx \sup _{b, w \in \mathbb{D}} \frac{|b|^{q}\left(1-|w|^{2}\right)^{1-\lambda}\left(1-|b|^{2}\right)^{\tau q}}{|1-\bar{b} \varphi(w)|^{q}} \int_{\mathbb{T}}\left|\frac{\varphi(\zeta)-\varphi(w)}{(1-\bar{b} \varphi(\zeta))}\right|^{q}\left|\sigma_{w}^{\prime}(\zeta)\right||\mathrm{d} \zeta| \\
& \gtrsim \sup _{w \in \mathbb{D}} \frac{|\varphi(w)|^{q}\left(1-|w|^{2}\right)^{1-\lambda}}{\left(1-|\varphi(w)|^{2}\right)^{q(1-\tau)}} \int_{\mathbb{T}}\left|\sigma_{\varphi(w)} \circ \varphi\right|^{q}\left|\sigma_{w}^{\prime}(\zeta)\right||\mathrm{d} \zeta| \\
& \approx \sup _{w \in \mathbb{D}} \frac{|\varphi(w)|^{q}\left(1-|w|^{2}\right)^{1-\lambda}}{\left(1-|\varphi(w)|^{2}\right)^{q(1-\tau)}}\left\|\sigma_{\varphi(w)} \circ \varphi \circ \sigma_{w}\right\|_{q}^{q},
\end{aligned}
$$

and hence the desired implication follows.

4.2. Actions between analytic Campanato and Bloch type spaces. The previous discussion leads to a consideration of the actions of $C_{\varphi}$ sending $\mathcal{A} \mathcal{L}_{p, \eta}$ to $\mathcal{B}_{\alpha}$ and its converse. To do so, we need the following existence result.

Lemma 13. For $0<\alpha<\infty$, there are two functions $f_{1}, f_{2}$ in $\mathcal{B}_{\alpha}$ such that

$$
\left(1-|z|^{2}\right)^{2 \alpha}\left(\left|f_{1}^{\prime}(z)\right|^{2}+\left|f_{2}^{\prime}(z)\right|^{2}\right) \approx 1 \quad \forall \quad z \in \mathbb{D} .
$$

Proof. This follows immediately from the inequalities (2.2) and (2.4) in the argument for [26, Theorem 2.1.1].

Below is an alternative to Theorem 1 (iv).

Theorem 14. Let $0<\eta<1+p<\infty, 0<\alpha, p-1<\infty$ and $\varphi$ be an analytic self-map of $\mathbb{D}$.

(i)

if and only if

$$
\left\|C_{\varphi} f\right\|_{\mathcal{B}_{\alpha}} \lesssim\|f\|_{\mathcal{A} \mathcal{L}_{p, \eta, \star}} \quad \forall \quad f \in \mathcal{A} \mathcal{L}_{p, \eta}
$$

$$
\sup _{w \in \mathbb{D}} \frac{\left(1-|w|^{2}\right)^{\alpha}\left|\varphi^{\prime}(w)\right|}{\left(1-|\varphi(w)|^{2}\right)^{\frac{p+1-\eta}{p}}}<\infty
$$

if and only if

$$
\left\|C_{\varphi} f\right\|_{\mathcal{A L}_{p, \eta, \star}} \lesssim\|f\|_{\mathcal{B}_{\alpha}} \quad \forall \quad f \in \mathcal{B}_{\alpha}
$$

$$
\sup _{I \subseteq \mathbb{T}}\left(\frac{1}{|I|^{\eta}} \int_{I}\left(\int_{1-|I|}^{1}\left|\varphi^{\prime}(r \zeta)\right|^{2}(1-r)^{1-2 \alpha} \mathrm{d} r\right)^{\frac{p}{2}} \frac{|\mathrm{d} \zeta|}{2 \pi}\right)^{\frac{1}{p}}<\infty .
$$


Proof. (i) The growth of functions in $\mathcal{A L}_{p, \eta}$ presented in the beginning of Section 3 derives that if $f \in \mathcal{A L}_{p, \eta}$ then

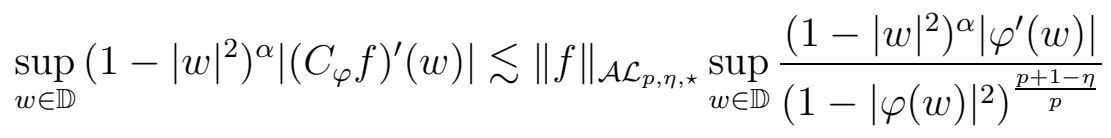

and hence the sufficiency of (i) is true. The necessity of (i) just follows from a simple calculation with a given point $w \in \mathbb{D}$ and the test function in $\mathcal{A} \mathcal{L}_{p, \eta}$ below:

$$
f_{w}(z)=\frac{\left(1-|\varphi(w)|^{2}\right)^{(p+\eta-1) / p}}{1-\overline{\varphi(w)} z} \quad \forall \quad z \in \mathbb{D} .
$$

(ii) The sufficiency can be checked by using the definition of $\mathcal{B}_{\alpha}$ and Theorem 5 (ii). Concerning the necessity, we utilize those two $\mathcal{B}_{\alpha}$ functions $f_{1}, f_{2}$ in Lemma 13 and the elementary estimate

$$
U^{p / 2}+V^{p / 2} \approx(U+V)^{p / 2} \quad \forall \quad U, V \geq 0
$$

to get that if

$$
\left\|C_{\varphi} f\right\|_{\mathcal{A L}_{p, \eta, \star}} \lesssim\|f\|_{\mathcal{B}_{\alpha}} \quad \forall \quad f \in \mathcal{B}_{\alpha}
$$

then

$$
\begin{aligned}
& \infty>\left\|f_{1}\right\|_{\mathcal{B}_{\alpha}}^{p}+\left\|f_{2}\right\|_{\mathcal{B}_{\alpha}}^{p} \\
& \gtrsim\left\|C_{\varphi} f_{1}\right\|_{\mathcal{A L}_{p, \eta, \star}}^{p}+\left\|C_{\varphi} f_{2}\right\|_{\mathcal{A} \mathcal{L}_{p, \eta, \star}}^{p} \\
& \gtrsim|I|^{-\eta} \int_{I}\left(\int_{1-|I|}^{1}\left|\left(f_{1} \circ \varphi\right)^{\prime}(r \zeta)\right|^{2}(1-r) \mathrm{d} r\right)^{\frac{p}{2}}|\mathrm{~d} \zeta| \\
&+|I|^{-\eta} \int_{I}\left(\int_{1-|I|}^{1}\left|\left(f_{2} \circ \varphi\right)^{\prime}(r \zeta)\right|^{2}(1-r) \mathrm{d} r\right)^{\frac{p}{2}}|\mathrm{~d} \zeta| \\
& \gtrsim|I|^{-\eta} \int_{I}\left(\int_{1-|I|}^{1}\left(\left|\left(f_{1} \circ \varphi\right)^{\prime}(r \zeta)\right|^{2}+\left|\left(f_{2} \circ \varphi\right)^{\prime}(r \zeta)\right|^{2}\right)(1-r) \mathrm{d} r\right)^{\frac{p}{2}}|\mathrm{~d} \zeta| \\
& \gtrsim|I|^{-\eta} \int_{I}\left(\int_{1-|I|}^{1}\left|\varphi^{\prime}(r \zeta)\right|^{2}(1-r)^{1-2 \alpha} \mathrm{d} r\right)^{\frac{p}{2}}|\mathrm{~d} \zeta|
\end{aligned}
$$

holds any subarc $I \subseteq \mathbb{T}$. This implies the desired inequality.

\section{REFERENCES}

[1] N. Arcozzi, D. Blasi and J. Pau, Interpolating sequences on analytic Besov type spaces, Indiana Univ. Math. J. 58(2009)1281-1318.

[2] S. Campanato, Proprietá di hölderianitá di alcune classi di funzioni, Ann. Scuola Norm. Sup. Pisa (3) 17(1963)175-188.

[3] S. Campanato, Proprietá di una famiglia dispazi funzionali, Ann. Scuola Norm. Sup. Pisa (3)18(1964)137-160.

[4] C. Cascante, J. Fàbrega and J. Ortega, The corona theorem in weighted Hardy and Morrey spaces, Ann. Scuola Norm. Super. Pisa Cl. Sci. DOI: 10.2422/2036-2145.201202-006.

[5] C.C. Cowen and B.D. MacCluer, Composition Operators on Spaces of Analytic Functions. Studies in Advanced Mathematics. CRC Press, Boca Raton, FL, 1995.

[6] X. Duong, J. Xiao and L. Yan, Old and new Morrey spaces with heat kernel bounds, J. Fourier Anal. Appl. 13(2007)87-111. 
[7] E. B. Fabes, R. L. Johnson and U. Neri, Spaces of harmonic functions representable by Poisson integrals of functions in $B M O$ and $\mathcal{L}_{p, \lambda}$, Indiana Univ. Math. J. 25(1976)159-170.

[8] C. Fefferman and E. Stein, $H^{p}$ spaces of several variables, Acta Math. 129(1972)137-193.

[9] P. G. Ghatage, and D. Zheng, Analytic functions of bounded mean oscillation and the Bloch space, Integral Equ. Oper. Theory 17(1993)501-515.

[10] M. Giaquinta, Multiple Integrals in the Calculus of Variations and Nonlinear Elliptic Systems, Ann. Math. Studies 105, Princeton University Press, Princeton, N.J., 1983.

[11] F. Jafari, B. MacCluer, C. Cowen and D. Porter (eds.), Studies on composition operators. Contemp. Math. 213, Amer. Math. Soc., 1998.

[12] F. John and L. Nirenberg, On functions of bounded mean oscillation, Comm. Pure Appl. Math. 14(1961)415-426.

[13] J. Laitila, Weighted composition operators on BMOA, Comput. Methods Funct. Theory 9(2009)2746.

[14] G. N. Meyers, Mean oscillation over cubes and Hölder continuity, Proc. Amer. Math. Soc. 15(1964)717-721.

[15] C. B. Morrey, On the solutions of quasi-linear elliptic partial differential equations, Trans. Amer. Math. Soc. 43(1938)126-166.

[16] J. Ortiz and A. Torchinsky, On a mean value inequality, Indiana Univ. Math. J. 26(1977)555-566.

[17] J. Peetre, On convolution operators leaving $L^{p, \lambda}$ spaces invariant, Ann. Mat. Pura Appl. (4)72(1966)295-304.

[18] J. Peetre, On the theory of $\mathcal{L}^{p, \lambda}$ spaces, J. Funct. Anal. 4(1969)71-87.

[19] J. H. Shapiro, Composition Operators and Classical Function Theory. Springer, New York, 1993.

[20] R. K. Singh and J. S. Manhas, Composition Operators on Function spaces. North-Holland Mathematics Studies, 179. North-Holland Publishing Co., Amsterdam, 1993.

[21] W. Smith, Composition operators between Bergman and Hardy spaces, Trans. Amer. Math. Soc. 348(1996)2331-2348.

[22] G. Stampacchia, $\mathcal{L}^{(p, \lambda)}$-spaces and interpolation, Comm. Pure Appl. Math. 17(1964)293-306.

[23] A. Torchinsky, On a mean value inequality, Bull. Amer. Math. Soc. 81(1975)950-953.

[24] A. Torchinsky, Real-variable Methods in Harmonic Analysis. Volume 123 in Pure and Applied Mathematics, Academic Press, 1986.

[25] Z. Wu and C. Xie, $Q$ spaces and Morrey spaces, J. Funct. Anal. 201(2003)282-297.

[26] J. Xiao, Holomorphic Q Classes, LNM 1767 Springer-Verlag, 2001.

[27] J. Xiao, Geometric $Q_{p}$ Functions, Birkhauser-Verlag, 2006.

[28] J. Xiao and W. Xu, Composition operators between analytic Campanato spaces, J. Geometric Anal. DOI 10.1007/s12220-9349-6.

[29] R. H. Zhao, Distances from Bloch functions to some Möbius invariant spaces, Ann. Acad. Sci. Fenn. Math. 33(2008)303-313.

[30] K. Zhu, Operator Theory in Function Spaces, American Mathematical Society, 2007.

[31] C. T. Zorko, Morrey Space, Proc. Amer. Math. Soc. 98(1986)586-592.

Jie Xiao, Department of Mathematics and Statistics, Memorial University, St. John's, NL A1C 5S7, CANADA

E-mail address: jxiao@mun.ca

Cheng Yuan, Institute of Mathematics, School of Science, Tianjin University of TECHNOlOGY AND EduCATiOn TIANJin 300222, ChinA

E-mail address: yuancheng1984@163.com 